\title{
Emerging organic contaminant removal in constructed wetlands
}

\author{
Haijing Ding ${ }^{1, a}$, Yanli Ding ${ }^{2, b}$ and Shaoyuan Bai ${ }^{3, c}$ \\ ${ }^{1}$ Guangxi Key Laboratory of Environmental Pollution Control Theory and Technology, Guilin \\ University of Technology, Guilin 541004, China \\ ${ }^{2}$ College of Earth Sciences, Guilin University of Technology, Guilin 541004, China \\ ${ }^{3}$ Collaborative Innovation Center for Water Pollution Control and Water Safety in Karst Area, Guilin \\ University of Technology, Guilin 541004, China \\ a913145045@qq.com, bdyl@glut.edu.cn cbaisy@glut.edu.cn
}

\begin{abstract}
Keywords: emerging organic contaminants, constructed wetland, removal mechanisms, design parameters
\end{abstract}

Abstract. This paper presents a comprehensive review of the current state of research activities on the application of constructed wetlands for removing emerging organic contaminants (EOCs) from wastewater. The design parameters of the reported constructed wetlands including the physical configuration, vegetation species, and targeting subjects were summarized .The removal efficiencies under different conditions in the wetlands were evaluated. In addition, the importance of the three main components of constructed wetlands (substrate, plants and microbes) for emerging organic contaminant removal was analyzed to elucidate the possible removal mechanisms involved .There is a general consensus among many researchers that constructed wetlands hold great potential of being used as an alternative secondary wastewater treatment system for the removal of pharmaceuticals.

\section{Introduction}

The occurrence of emerging organic contaminants (EOCs), such as pharmaceutical and personal care products (PPCPs), pesticides or antiseptics in poorly treated wastewater and eventually in other watercourses constitutes nowadays an increasing concern worldwide due to their possible toxicological effects to the environment and living organisms ${ }^{[1]}$. One of the main sources of EOCs in the environment is the effluent from conventional wastewater treatment plants (WWTPs), where EOCs removal is often incomplete ${ }^{[2]}$. However, EOCs end up in vital aquatic compartments, such as surface water, ground water and even drinking water, at concentrations between few $\mathrm{ng} / \mathrm{L}$ and several $\mathrm{mg} / \mathrm{L}$, with negative impact on water quality. The occurrence of EOCs in the environment is reported in thousands of publications during the last decades and reviewed by many authors demonstrating an increasing concern about them ${ }^{[3]}$.Constructed wetlands $(\mathrm{CWs})$ are low-cost wastewater treatment options, which are able to remove or attenuate a variety of waterborne contaminants including $\mathrm{PPCPs}^{[4]}$. The removal of emerging organic contaminants (EOCs) by constructed wetlands (CWs) has been gaining more interest in the last decade ${ }^{[5]}$. However, it is still unclear which are the key factors influencing the removal process.

\section{Removal mechanisms of EOCs in CWs}

Pharmaceuticals and personal care products (PPCPs) in the aquatic environment are regarded as emerging contaminants and have attracted increasing concern. The removal of pharmaceuticals and PPCPs in CWs often involves a diverse and complex set of physical, chemical and biological processes, which can be affected by the design and operational parameters selected for treatment.

Removal of antibiotics in livestock wastewater can be achieved through a variety of degradation mechanisms, including physicochemical degradation, photo degradation (photolysis), biodegradation (microbial activity), and adsorption in wetland soil and plants (available organic surfaces). The removal mechanisms generally depend on the physicochemical properties of antibiotics ${ }^{[6]}$. 
Constructed wetlands (CWs) are low-cost wastewater treatment options, which are able to remove or attenuate a variety of waterborne contaminants including PPCPs. However, due to the large surface area per inhabitant needed to reach the target water quality parameters, their implementation is only feasible in small urban communities or as tertiary treatments dealing with a small, diverted fraction of conventional WWTP effluents behavior of EOCs in constructed wetland compartments: Influent, effluent, pore water, substrate and plant roots ${ }^{[7]}$.

The mechanisms involved in pollutant removal in CWs can be classified into biotic processes (e.g. microbiological degradation, biofilm, root and plant uptake) and physicochemical processes (evaporation, photo degradation, oxidation, hydrolysis, retention or root sorption into the gravel bed). Nevertheless, CWs are highly complex systems presenting several environments and microenvironments, where different physical-chemical conditions can reign, influencing the various above mentioned removal processes. These different environments inside the system depend on the specific CW design, namely, pore water, upper layer exposed to the sunlight, plants, biofilm on the substrate, and roots' biofilm .Therefore, due to this complexity, removal mechanisms in CWs are not fully understood yet ${ }^{[4]}$.

\section{influence of main CW design parameters}

\section{Influence of vegetation}

The relative importance of the presence of plants and the ability of particular species to improve the removal efficiency of EOCs in CWs is still unclear. This is because many other factors, namely their related microorganism communities and the nature of the wastewater, as well as environmental and operational conditions may all be acting in concert. Indeed, the presence of plants improves the degradation of naproxen, ibuprofen, diclofenac and caffeine, with $P$. australis displaying better performance than Typha, at least in the summer months ${ }^{[8]}$.

Various authors have postulated that more developed aerial and underground parts of plant species can improve the removal of EOCs in a CW. Indeed, a slight difference in acetaminophen removal between two H-SSF beds planted with $\quad P$. australis and Typha has been documented, although this difference was not statistically significant at the $95 \%$ confidence level $^{[8]}$.

\section{Influence of primary treatment}

Various primary treatments have been studied and included in many papers. In particular, some authors compared the performance of a primary clarifier with an anaerobic hydrolysis up flow sludge bed (HUSB). These systems produce effluents of differing redox potential and dissolved oxygen concentrations, which may affect processes occurring within the $\mathrm{CWs}^{[9]}$. Specifically, the sequence fed with primary clarifier effluent exhibited slightly higher removal efficiencies for ketoprofen and ibuprofen than that fed by HUSB effluent.

\section{Influence of loading mode}

Some papers observed a considerable enhancement in EOCs removal, for ibuprofen, diclofenac and naproxen, when H-SSFCWs were fed in batch mode. This they ascribed to the higher redox status caused by alternating cycles of saturation and unsaturation ${ }^{[10]}$. In contrast, the differences between batch and continuous loading were very small.

\section{Influence of HRT}

As previously mentioned, HRT is a key parameter in EOCs removal in $\mathrm{CWs}^{[11]}$. The greater the HRT, the higher the removal efficiencies for most of the selected compounds, in particular hydrophobic compounds such as hormones. Significant correlations $(p<0.05)$ were also found between HRT and the removal of caffeine, salicylic acid, ketoprofen and clofibric acid, and they reported Pearson correlation values of 0.99 for all 4 compounds ${ }^{[12]}$.

Influence of specific surface area $\left(\mathrm{m}^{2} / \mathrm{PE}\right)$

An interesting parameter to use in comparison between the performances of different CWs is the specific surface area defined as the ratio between the area of a single step and the number of PE corresponding to the applied hydraulic load (assuming a specific water consumption per person per 
day) ${ }^{[13]}$. Unfortunately, this parameter has only been evaluated in a few cases and data is difficult to interpret, as it all refers to the surface of the whole treatment line and not to the specific step under evaluation $^{[14]}$.

\section{Problems and Prospect}

For the treatment of pharmaceutical contaminants in wastewater using constructed wetlands, it is really a fresh application field. The feasibility of constructed wetlands to eliminate pharmaceuticals in wastewater is requiring comprehensive understanding on the removal efficiencies, the removal mechanisms, the influences of design and environmental factors, and the toxicity risks ${ }^{[12]}$. Hence, much more attention is needed to pay for these issues in future research studies.

Due to the environmentally-friendly nature of a $\mathrm{CW}$, more researchers and engineers are investigating their suitability for removing EOCs from wastewater. Physicochemical reactions responsible for the removal of antibiotics flowing in a $\mathrm{CW}$ are rather well documented. However, it has not been investigated in depth what microorganisms are responsible for the EOCs removal in a $\mathrm{CW}^{[15]}$. In addition, the way in which EOCs are developed or reduced in a $\mathrm{CW}$ has not been sufficiently explored; the operating conditions under which EOCs are developed or reduced needs to be studied further. In particular, the relationship between the flow scheme of a $\mathrm{CW}$ and the abundance of EOCs should be explored. Nanoparticles are entering into the wastewater effluent and sludge, but their influence on the EOCs is largely unknown. Future research may include studies of the effect of nanomaterials on the mechanism of transfer of EOCs in order to evaluate viability of ARB and residual copy numbers of EOCs.

Current knowledge is limited on the removal efficiencies of EOCs in constructed wetlands, the removal mechanisms involved, the toxicity to constructed wetlands caused by EOCs, and the influences of certain important parameters (configuration design, hydraulic mode, temperature and seasonality, $\mathrm{pH}$, oxygen and redox potential, etc.). Further research on these issues to provide more and better convincing evidences for the function and performance of the constructed wetlands are needed.

\section{Acknowledgements}

This work was funded by the National Natural Science Foundation of China (No. 51638006, 41404116), Guangxi Natural Science Foundation (No. Guikehe1599005-2-2), and was supported by Guangxi Scientific Experiment Center of Mining, Metallurgy and Environment and the project of high level innovation team and standing scholar in Guangxi colleges and universities.

\section{References}

[1] Ávila, C.,J.M. Bayona , I. Martín. Emerging organic contaminant removal in a full-scale hybrid constructed wetland system for wastewater treatment and reuse[J].Ecological Engineering,2015.80:108-116.

[2] Avila, C.,J. Nivala,L. Olsson. Emerging organic contaminants in vertical subsurface flow constructed wetlands: influence of media size, loading frequency and use of active aeration[J].Sci Total Environ,2014.494-495:211-7.

[3] Barbosa, M.O.,N.F. Moreira,A.R. Ribeiro. Occurrence and removal of organic micropollutants: An overview of the watch list of EU Decision 2015/495[J].Water Res,2016.94:257-79.

[4] Hijosa-Valsero, M.,C. Reyes-Contreras,C. Dominguez. Behaviour of pharmaceuticals and personal care products in constructed wetland compartments: Influent, effluent, pore water, substrate and plant roots[J].Chemosphere,2016.145:508-17. 
[5] Zhang, Y.,T. Lv,P.N. Carvalho. Ibuprofen and iohexol removal in saturated constructed wetland mesocosms [J].Ecological Engineering,2017.98:394-402.

[6] Choi, Y.-J.,L.-H. KimK.-D. Zoh. Removal characteristics and mechanism of antibiotics using constructed wetlands[J].Ecological Engineering,2016.91:85-92.

[7] Matamoros, V.,E. Uggetti,J. Garcia. Assessment of the mechanisms involved in the removal of emerging contaminants by microalgae from wastewater: a laboratory scale study[J].J Hazard Mater,2016.301(2016):197-205.

[8] Verlicchi, P.E. Zambello. How efficient are constructed wetlands in removing pharmaceuticals from untreated and treated urban wastewaters? A review[J].Sci Total Environ,2014.470-471:1281-306.

[9] Zhang, D.,R.M. Gersberg,W.J. Ng. Removal of pharmaceuticals and personal care products in aquatic plant-based systems: a review[J].Environ Pollut,2014.184:620-39.

[10] Fountoulakis, M.S.,G. Sabathianakis,I. Kritsotakis. Halophytes as vertical-flow constructed wetland vegetation for domestic wastewater treatment[J].Science of the Total Environment,2017.583:432-439.

[11] Fischer, A.,M. ManefieldP. Bombach. Application of stable isotope tools for evaluating natural and stimulated biodegradation of organic pollutants in field studies[J].Curr Opin Biotechnol,2016.41:99-107.

[12] Li, Y.,G. Zhu,W.J. Ng.A review on removing pharmaceutical contaminants from wastewater by constructed wetlands: design, performance and mechanism[J].Sci Total Environ,2014.468-469:908-32.

[13] Lv, T.,Y. Zhang,L. Zhang. Removal of the pesticides imazalil and tebuconazole in saturated constructed wetland mesocosms[J].Water Res,2016.91:126-36.

[14] Matamoros, V.,Y. RodriguezJ. Albaiges. A comparative assessment of intensive and extensive wastewater treatment technologies for removing emerging contaminants in small communities[J].Water Res,2016.88:777-85.

[15] Sharma, V.K.,N. Johnson,L. Cizmas. A review of the influence of treatment strategies on antibiotic resistant bacteria and antibiotic resistance genes[J].Chemosphere,2016.150:702-14. 